Alain Rabatel et Solveig Lepoire

IUFM de Lyon, ICAR, U.M.R. C.N.R.S. 5191,

Université Lumière-Lyon 2

Alain.Rabatel@ens-lsh.fr

lepoire-duc@wanadoo.f

\title{
Le dialogisme des discours représentés et des points de vue dans les explications, entre concordance et discordance
}

Cet article se propose d'examiner comment le dialogue en interaction influe sur l'organisation de la séquence explicative, sur ses enjeux cognitifs ou sur la gestion des interactions. Il s'agira de vérifier si, et si oui, comment les formes d'hétérogénéité, qui participent de la discordance énonciative, sont malgré tout en capacité de participer à une certaine " concordance » cognitive et interactionnelle. Pour ce faire, on s'attachera notamment à la place et au rôle du discours représenté (DR) dans l'économie générale du type de texte explicatif, ainsi qu'aux postures énonciatives qu'il construit.

Compte tenu du genre retenu, celui des explications en contexte didactique interactionnel, deux modèles de l'explication sont convoqués conjointement ou alternativement, l'un, comme mise en discours d'un savoir préalable construit et élaboré (c'est l'explication "classique »), l'autre comme démarche tâtonnante de coconstruction d'une explication [I]. Ces deux modèles sont activés le plus souvent par des acteurs différents, le maître privilégiant le premier, les élèves le second. Les formes de DR utilisées par les locuteurs et leur fonction varient selon les interactants [2]. Chez le maître, auquel nous consacrerons l'essentiel de nos analyses, le DR est le plus souvent mis à contribution pour co-construire un point de vue (PDV) dans des interactions dissymétriques. L'enseignant privilégie tantôt une posture de surénonciateur surplombant grâce au DR par autorité polyphonique ou à des coups de force qui tirent 
abusivement la parole des élèves du côté du Savoir, pour débloquer une interaction qui s'enlise, tantôt une posture de sousénonciateur ${ }^{\mathrm{I}}$, donnant la parole de façon distanciée à un élève imaginaire réduit à un rôle d'expérimentateur ou d'explicateur naïf, permettant ainsi aux élèves d'objectiver et de rectifier eux-mêmes ce qui est erroné dans le discours qui leur est imputé. Ainsi, les phénomènes d'hétérogénéité énonciative, en dépit de leur diversité et de leur dissymétrie, co-construisent des PDV en mouvement perpétuel, au cours de processus langagiers en tension, entre « discordance concordante » et " concordance discordante " - notions que nous empruntons à Ricœur et transposons dans le contexte spécifique de l'explication [3].

\section{Le type de texte explicatif}

On laissera de côté la discussion des relations entre les types de texte explicatif et informatif, et l'on considèrera l'explication comme un type de texte (ou une séquence macro-textuelle) qui se manifeste chaque fois que le locuteur anticipe un problème de communication, de compréhension, ou répond à une difficulté de cet ordre qui s'est manifestée dans l'interaction (Bronckart I997).

I.I. Sur le plan idéationnel et conceptuel, l'explication consiste dans l'examen des relations entre un phénomène à expliquer (explanandum ou explicandum) et un phénomène expliquant (explanans ou explicans), qui ne sont en principe pas du même niveau, ou plutôt, dont la nature de la relation est fonction des genres de discours : ainsi, une explication didactique ne partage pas les mêmes routines qu'une explication scientifique devant des pairs. Mais, que l'explication soit fonctionnelle, intentionnelle ou causale, il n'est pas facile de la définir clairement d'un point de vue général, ainsi que le reconnaît Plantin qui définit le concept comme

une activité cognitive, langagière, interactionnelle, déclenchée par le sentiment ou l'expression d'un doute, d'une ignorance, d'un trouble

I. Cf. Rabatel 2002, 2004a, b, c, 2005 et infra, 3, pour une approche plus détaillée de la co-, sur- et sousénonciation ainsi que des notions de concordance et de discordance. 
dans le cours normal de l'action ou d'un simple malaise («mental discomfort», Wittgenstein I975:26). L'explication est ce discours ou cette interaction qui satisfont un besoin cognitif, apaisent un doute et produisent un sentiment de compréhension (Plantin 2002 : 253).

Dans tous les cas, l'explication révèle un aspect (du fonctionnement) du monde, et c'est ce qui la distingue de l'explicitation, qui ne redit que ce qui a été dit (reformulation, glose, etc.).

La Segmented Discourse Representation Theory distingue 1'Explication de l'Élaboration, du Résultat, de l'Arrière-Plan et de la Narration. Selon de Saussure 2003, l'Explication seule relève d'un ordre temporel inverse à l'ordre temporel de la Narration : ainsi, dans

Max est tombé (a). Paul l'a poussé (b),

l'éventualité (a) s'est produite après l'éventualité (b), alors que dans la Narration, l'ordre des éventualités racontées correspond à celui des événements. De même, dans le Résultat, l'élément résultatif suit la mention de l'élément causant, contrairement à ce qui se passe dans l'Explication. Enfin, l'Explication diffère de l'ArrièrePlan en ce qu'elle relève d'une éventualité qui existe avant l'éventualité résultative (et pas pendant son déroulement) tandis que l'éventualité d'Arrière-Plan existe avant et pendant l'éventualité résultative, comme dans l'Arrière-Plan de (2) (Pierre a mal au poumon avant et après le diagnostic) à la différence de l'explication de (3), où l'exposition au soleil est antérieure au coup de soleil, mais ne dure pas :

(2) Pierre a la tuberculose. Il avait mal au poumon.

(3) Pierre a la peau brûlée. Il s'était longtemps exposé au soleil I.

La visée formelle de la SDRT contraint à réfléchir sur des paires d'énoncés fabriqués (ou attestés), en fonction d'une conception de l'explication comme réponse incontestée à un problème circonscrit : les représentations sous-jacentes diffèrent des conceptions processuelles de l'explication comme co-construction, dans des séquences

I. Exemples (2) et (3) empruntés à Martin 2005. 
interactionnelles plus longues que les paires d'énoncés précédentes (cf. infra, I.4).

I.2. Sur le plan structurel, les dimensions cognitives et interactionnelles donnent lieu à des structures différentes selon l'importance accordée à telle composante. La structure tripartite du type de texte explicatif comporte trois phases, cognitives et interactionnelles, chez Coltier I 986, avec phases de questionnement, de résolution et de conclusion (comme chez de Gaulmyn et Gülich) :

Phase I : constitution d'un objet à expliquer, des rôles de sujet qui explique et de sujet auquel s'adresse l'explication et/ou qui la reçoit;

Phase 2 : noyau explicatif;

Phase 3 : ratification et clôture de la séquence sur un « consensus supposé obtenu au terme de l'explication».

La structure duelle privilégie les phases cognitives de questionnement et de résolution, autour des schématisations des objets du discours qui entourent les opérateurs, pourquoi $P$ et parce que $Q$ (Grize I990) :

Schématisation initiale POURQUOI P Sch. du problème PARCE QUE Q Sch. explicative Objet complexe $>>>>>>>$ Problème $>>>>>>$ Explication (Grize I990)

Ces schématisations se retrouvent chez Adam 2005, complétées par des paramètres interactionnels :

\begin{tabular}{|llll|}
\hline & P. explicative 0: & schématisation initiale [description] \\
Pourquoi $P ?$ & P. explicative I : & Problème (question) \\
Parce que $Q$ & P. explicative 2: & Explication (réponse) \\
& P. explicative 3: & Ratification-évaluation (Adam 2005: I67) \\
\hline
\end{tabular}

Les représentations précédentes ont été élaborées à partir de contextes monologaux, le plus souvent dans des textes écrits, plus rarement à l'oral - comme quand un locuteur s'explique à luimême un problème quelconque (de Gaulmyn I99I) : dans ces contextes, pourquoi $P$ et parce que $Q$ sont pris en charge par le 
même locuteur, selon un mouvement dialogique interne à l'énoncé. En contexte dialogal, pourquoi $P$ est pris en charge par un locuteur, et parce que $Q$ par un autre (Joab et Rossari I999, Bruxelles et de Gaulmyn 200I). Une des hypothèses du présent travail sera de voir dans quelle mesure le contexte dialogal démultiplie et complexifie le dialogisme lors du processus de co-construction des explications.

I.3. Sur le plan de la mise en discours, les diverses phases de l'explication « n'apparaissent pas nécessairement » dans l'ordre des représentations abstraites précédentes, " et la phase de questionnement ne contient pas obligatoirement une interrogative indirecte $»(\mathrm{Col}-$ tier I $986: 8$ ). Néanmoins, on rencontre fréquemment un pourquoi, associé à un parce que, lui-même entouré d'un présentatif et de locutions prépositives ou conjonctives - c'est parce que, c'est pour que, c'est en raison de, c'est que, cela tient à (Adam 2005: I62-I65) -, ou des structures rétroactives en c'est pourquoi, voilà pourquoi, qui ont une valeur présentative et représentative (Rabatel 2000 et 200 I) intensifiant l'explication, renvoyant au co-texte antérieur comme à la situation, associant de ce fait fort efficacement le coénonciataire au raisonnement explicatif. Mais ces marques ${ }^{I}$ relèvent plutôt de l'explication causale (en pourquoi), souvent privilégiée, que de l'explication procédurale (en comment), mettant à contribution d'autres marques, notamment les marqueurs spatio-temporels qui planifient les segments descriptifs et narratifs balisant le parcours explicatif.

La présence de cette structure et de ces marques permet de distinguer, sur le plan linguistique, la forme explicative de la fonction explicative, qui peut être éventuellement assurée par d'autres types de textes, par exemple des récits, des informations, des descriptions ou des argumentations. Sur un plan discursif, le type de texte explicatif peut ainsi comporter des enchâssements de textes informatifs, descriptifs, narratifs ou argumentatifs ${ }^{2}$ qui jouent une fonction explicative sans relever pour autant du type de texte explicatif - comme

I. Colletta 2004: 236 souligne que ces marques sont de plus en plus nombreuses et d'un usage diversifié avec l'âge.

2. Plantin souligne que les lois de passage entre explanans et explanandum sont les mêmes pour l'argumentation et l'explication, à cela près que dans l'explication, l'explanandum est avéré et qu'il faut trouver l'explanans (Plantin 2002 : 253-254). 
il peut lui-même être enchâssé dans chacun des types de texte précédents. Les différents moments de l'explication sont souvent corrélés à des plans d'énonciation distincts, avec énonciation impersonnelle dans les descriptions, énonciation personnelle dans les énoncés balises qui donnent le plan de texte de l'explication, et, dans la partie proprement informative, des énoncés avec effacement énonciatif ou implication de l'énonciateur et du coénonciataire dans les cas où l'auteur de l'explication sent ou veut montrer les problèmes ou les enjeux de telle ou telle explication (Coltier I 986 : 9-Io).

I.4. Selon d'aucuns, les conditions pragmatiques de recevabilité de l'explication impliqueraient qu'elle soit objective, déconnectée de l'action et que son auteur soit «autorisé » et évite toute polémique ${ }^{\mathrm{I}}$. Le croisement de cette thèse, d'inspiration bourdieusienne (Ebel I98 I : I 8-I 9, 26), avec la distinction entre explication et justification (d'inspiration piagetienne ${ }^{2}$ ), restreint l'explication au seul domaine des faits scientifiques reconnus et limite le statut d'explicateur au savant, qui serait seul légitime. On est loin des représentations spontanées ou ordinaires dans lesquelles l'explication se co-construit au fil de l'interaction, loin aussi des explications scientifiques en contexte didactique qui font l'objet de débats où la justification et l'argumentation ont une large place. À l'instar de Morf et Leclerc I 98 I, Moirand 2002 : 256, nous n'envisagerons pas ici l'explication que comme un transfert de connaissances visant une certaine généralité 3 , mais comme un processus qui fait passer d'un état cognitif insatisfaisant à un état plus satisfaisant - processus qui n'écarte pas la possibilité d'user d'arguments (Plantin 2002 : 254). Cela souligne l'importance de la distinction entre explication, comme mise en discours d'un texte préalablement construit, et démarche explicative, comme élaboration de connaissances (Miéville I98I : I25ss), ainsi que le confirment nos différents corpus, le maître privilégiant

I. En opposition à cette thèse normative, Plantin rappelle à propos qu'une même question en pourquoi, qui appelle une explication, dans une situation didactique inégale, peut se transformer en une argumentation si elle est traitée par un expert face à ses pairs (Plantin $2002: 254$ ).

2. Cf. l'opposition piagetienne reprise par Grize (I98I : 8) et Borel (I98I : 66) : l'explication causale est basée sur les rapports entre les choses, indépendamment du point de vue de l'énonciateur, contrairement à la justification.

3. Cf. supra, I.I. à propos de la SDRT. 
plutôt ${ }^{\mathrm{I}}$ le modèle de l'explication causale et les élèves celui du parcours explicatif avec des explications procédurales.

\section{Variété des formes de discours représentés et de dialogisme dans l'explication}

On considèrera que le discours représenté (DR) - que, pour des raisons énonciatives et pragmatiques nous préférons nommer ainsi plutôt que discours rapporté (cf. Nølke et al. $2004: 57$ et Rabatel 2003a) - est un sous-ensemble de la problématique plus vaste du dialogisme (Bres et Verine 2002 : I 59).

L'approche dialogique du DR prend en compte un large éventail de formes : DD, DI, DIL, discours direct libre, discours narrativisé (DN), îlots textuels de DD, DD avec que, formes de modalisation d'emprunt en selon - ou un équivalent, cf. l'exemple (I I) de modalisation autonymique d'emprunt ( il fera "un temps de rêve" comme dit X», cf. Authier-Revuz), conditionnel d'altérité énonciative qu'on rencontre dans les hypothèses, et pas seulement sous la plume des journalistes, comme en (4), (I5) ou (I6). Ces formes sont dialogiques dans la mesure où le discours de l'un est truffé de traces des PDV des autres par hétérodialogisation interlocutive (anticipation d'un à dire dans la situation d'interlocution), hétérodialogisation interdiscursive (écho d'un déjà dit dans l'interdiscours), ou encore par autodialogisation (elle aussi interlocutive ou interdiscursive), etc.

\section{I. Diversité des marques du discours représenté dans l'explication}

Listons rapidement quelques unes de ces marques et précisons leur lieu d'émergence dans la séquence explicative, avant d'analyser plus en détail leur fonction énonciative dans le cadre de l'explication. On rencontre de fréquents fragments de DD ou des îlots textuels au DD (en gras) :

I. Nous soulignons une tendance, sans prétendre forcer le lien entre telle pratique et tel locuteur, selon son statut et sa place, pas plus que nous ne voulons indiquer que l'explication se réduirait à l'explication causale, et le parcours explicatif à une explication procédurale. 
(4) (Corpus « Machin mouillé $\left.{ }^{~} »\right)$

M et et your shoes/ oo get o wetl et pourtant/ o Antoine y nous dit/ ben quan $[\mathbf{t}] \mathbf{y}$ en a deux : / ou quand c'est p-pluriel $/$ et ben : on devrait ${ }^{2}$ mettre un $s \backslash$ oo alors : qu'est-c 'que c'est qui : va pas Io

(5) (Corpus « Grenouilles »)

M si j’reprends ce que tu viens de dire / o Célia / o pour voir si on est bien d'accord / o quand la queue rap 'tissit les pattes arrières poussent $/$ oo et remplacent I ou alors c'est la queue qui se transforme en pattes $\backslash$

(6) (Corpus « Meité Paulo»)

PAU /ou bien/on : on fait un : titre à nous / o

MEI oui pff o on dit euh : devoirs à la maison/ point d'interrogation / ((petit rire))

I. Les corpus « Dinosaures », «OVNI » et «Grenouilles » ont été recueillis et transcrits par S. Lepoire, le corpus « Polyèdres » par M.-C. Guernier et V. DurandGuerrier, le corpus «Machin mouillé » par A. Pastor et A. Chalivet, le corpus «Méité Paulo» par R. Bouchard, M.-M. de Gaulmyn et A. Chalivet. La place nous manque pour préciser chacune des situations didactiques - mais cela ne nous semble pas peser fondamentalement sur les analyses. Pour de plus amples précisions, on se reportera à Lepoire I999 (« Ovni»), De Gaulmyn et alii $200 \mathrm{I}$ ( Meité Paulo»), Rabatel 2004a et d («machin mouillé »), Guernier et alii 2006 («Polyèdres ») et Lepoire, à paraître («Grenouilles »). On a cherché à unifier des transcriptions opérées dans des cadres théoriques distincts.

Conventions de transcriptions ICOR, version I.0.4 (avril 2005) :

$\mathrm{M}$

E

Es

STE

$=$

[]

$::$

0

1

I

dEUX

Man-

d'jà

((petit rire))

(inaud.)
Enseignant

Un élève non identifié

plusieurs élèves

Elève identifié

Enchaînement immédiat

Chevauchement

son allongé (:: répétés en fonction de la durée de l'allongement)

Pause intra-tour (oo répétés en fonction de la longueur de la pause)

Intonation descendante

Intonation montante

Intensité accrue

troncation

élision

description d'une production vocale

structure segmentale totalement inaudible

2. «Devrait» pourrait dans l'absolu être interprété comme un déontique, sans valeur d'altérité énonciative (et comme tel, il pourrait figurer dans les propos d'Antoine). Mais ce n'est pas le cas en contexte, cf. Rabatel 2004d. 
(7) (Corpus «OVNI»)

M euh:: alors moi je pense qu'y va pas falloir qu'on fasse comme ça $\backslash$ oo hein toi tu dirais:: $\mathbf{y}$ a des : des élèves qui vont faire $\mathbf{o}$ un compte rendu avec $\mathbf{o}$ toutes les idées pour $/$ [ et puis les autres qui font le compte rendu ] qu'avec les idées contrel

E [ non faut mélanger/faudrait mélanger les deux/]

(8) (Corpus « Machin mouillé »)

M ben on va l'réécouter/on va voir/ o alors on a plusieurs choses qui vont oo un peu/ on sait pas trop l

o on a dit / y en a qui disent you / o y en a qui disent your/oo y a un peu ça / le premier mot d'la phrase on sait pas trop/ on a un problème avec ça / oo y en a qui disent des fois get / o y en a qui disent des fois gets $\backslash$ oo alors on va vérifier on va écouter un p'tit peu, oo est-c'que ça change/ dans l'texte/ est- c'que c'est toujours pareil/ oo on va essayer d'regarder ça \d'écouter un peu $\backslash 000$ alors j'ai calé juste sur la fin \hein / à partir du moment où elle commence à être euh o bon elle a couru jusqu'à la maison / et on commence là $\ 000$

Les DD sont marqués par des changements de rythme, de hauteur et d'intonation aidant au repérage du changement d'énonciateur, comme en (5) où le DD est suivi par une reformulation par laquelle l'enseignant rapporte un PDV alternatif (cf. infra, (Io)). Ces variations jouent pour le repérage des mentions de DD en (8), le passage du français au code anglais étant par lui-même suffisamment explicite pour indiquer des reprises, renvoyant secondairement à des locuteurs distincts selon les réalisations phonétiques, sur lesquelles l'enseignant insiste, sur le plan intonatif, parce qu'elles sont la condition du raisonnement morphologique ultérieur (Rabatel 2004d). Cette double caractérisation, comme îlot (textuel) de DD et comme reprise, confirme l'intrication des phénomènes de prise en charge énonciative ${ }^{\mathrm{I}}$, et correspond à une situation que nous analyserons plus loin en terme de surénonciation : nous voulons dire par là que le locuteur citant, en insistant sur les différences phonologiques, qui étaient certes présentes chez les locuteurs cités, sans être aussi objectivées, cherche à faire comprendre à ses interlocuteurs qu'il y a là un problème, à charge pour eux de l'expliciter. Autrement dit, il y a là une posture surplombante, parce que le locuteur citant, tout en

I. Elle explique que nous parlions de discours représenté, plutôt que de discours rapporté, parce que même dans le cas du DD, ce dernier n'est jamais cité à l'identique et renvoie toujours aux motivations du locuteur citant. 
paraissant reprendre le discours de ses interlocuteurs, va plus loin qu'eux, même si son énoncé reste sibyllin.

Les exemples de DI (en italiques) sont peu fréquents dans nos corpus ${ }^{\text {I }}$ (cf. les corpus de Vincent et Dubois I $997: 47$, comptant 86\% de DD et $\mathrm{I} 4 \%$ de DI) :

(9) (Corpus « OVNI»)

M peut-être donc si on reprend un petit peu les idées hein Etienne y dit que : on peut parler du débat $\backslash$ d'un débat $\backslash$ parler des arguments pour : I des arguments contre : / o et puis euh : à la fin le pour et contre en conclusion/ ou y en a d'autres qui ont dit dans la conclusion mettre euh o son avis $\backslash$ o oui $\backslash \mathrm{o}$ Nadège /

(Io) (Corpus «Grenouilles»)

M si j’reprends ce que tu viens de dire / o Célia / o pour voir si on est bien d'accord / o quand la queue rap `tissit les pattes arrières poussent / oo et remplacent / ou alors c'est la queue qui se transforme en pattes $\backslash$ oo parce que lui il dit que c'est la queue qui se transforme en pattes $\backslash$

(I I) (Corpus «OVNI»)

M alors toi donc tu disais le débat il sert à rien parce que / o y'en au qui n'ont:: à ton avis personne n'a changé d'avis $\$ hein c'est ça /

E si

M alors $\backslash$ o vous levez le doigt pour répondre à Nadège / ooo tu disais que ça n'a servi a rien personne n'a changé d'avis I

La plupart des exemples antérieurs se caractérisent par le phénomène de bivocalité dialogique ${ }^{2}$, avec une superposition des voix et un entrelacement des PDV, comme l'indique la proximité des DR et de portions d'énoncés qui relèvent tantôt de reprises (à l'identique), tantôt de reformulations, lorsque le locuteur citant fait écho au PDV antérieur d'un interlocuteur, en le formulant dans ses propres mots et selon son propre PDV, en sorte qu'abondent les modalisations en discours second, cumulant le plus souvent un dit en usage et un dire en mention, comme c'était déjà le cas en (IO) et en (I I), et comme on le remarque plus nettement encore en (I 2) :

I. Nous n'avons pas trouvé d'exemples nets de discours narrativisé dans nos corpus sauf dans des phases de récapitulation qui ne sont pas en relation avec un contexte explicatif.

2. Cf. en (I I), le rôle de «à ton avis », qui, comme « selon », est une forme intermédiaire particulièrement efficace de discours bivocal (Rosier I999 : I89-I93) qui rapporte un dit du locuteur cité tout en étant de surcroît passible d'une explicitation de ce dit par le locuteur citant. 
(I2) (Corpus « Dinosaures »)

I E maître c'est pareil un météorite et un astéroïde

2 M bon oo alors est-ce que c'est différent un astéroïde et un météoritel ooo qui c'est qui dit oui qui c'est qui dit non pourquoi hein/ faut donner des arguments $\backslash$ o oui

3 LOI un astéroïde c'est un bout d'une planète et un météorite c'est::

4 M qui est:: d'un autre avis que:: Loïc et pourquoi\

5 E ben je sais pas

6 M Etiennel

7 ETI moi parce que:: un bout de un morceau d'une planète et pis : quelque chose qui se ballade dans le ciel c'est la même chose /

$8 \mathrm{M}$ alors à ton avis un astérö̈de et un météorite c'est la même chose I o qui est d'accord avec Etienne $\backslash$ o et pourquoi $\backslash$

9 E ben $\mathrm{j}$ 'sais pas

Io M qui n'est pas d'accord et pourquoi o Stéphane

I I STE moi parce un a : un astéroïde ça peut être plus gros qu'une météorite /

I2 M ah oo un astéroïde ça pourrait être plus gros qu'une météoritel

I3 EI ben oui parce que faut : parce que ils auraient pas appelé un:: un::

I4 E d'un différent nom /=

I5 EI =d'un différent nom ouais la même chose d'un différent nom parce si un astéroïde c'est un : un bout $\mathrm{d}$ : d'une planète c'est quand même gros / c'est quand même gros/ une planète \si un météorite c'est quelque chose qui se ballade dans l'espace c'est quand même [un météorite]

I6 M [d'accord bon/]

I7 E oui mais dans le film y parlaient des deux en même temps/=

I 8 M =voilà y disaient les deux mots hein dans le film $\mathbf{y}$ disent les deux mots nous on va dire comme ça $\backslash \mathrm{o}$ on va dire qu'un astérö̈de c'est un gros / o météorite \d'accord / o bon \o donc on va dire que c'est la même chose hein \

En effet, les modalisations du dire sont nombreuses, en (I2), notamment lorsque le maître reformule le point de vue de Stéphane en modalisant avec le conditionnel, pour faire entendre une distance propice à l'explication. De même dans le dernier tour de parole : la différence, pourtant entérinée comme vraie, n'est pas jugée pertinente par le maître, en fonction du niveau d'explication retenu pour la disparition des dinosaures ${ }^{\mathrm{I}}$. La prise en compte de la problématique des reformulations complexifie l'analyse des formes de DR : ainsi, en Io, le maître procède à une auto-reformulation de 8 ; de

I. Sur le fond, on peut discuter, mais tel semble être néanmoins le point de vue du maître. 
même en I8, «y disent les deux mots » reformule ce qui précède en changeant le tiroir verbal.

Nous n'exemplifierons pas davantage ces formes, nous les analyserons plus en détail en 2.2. et en 3. pour rendre compte du fait que le DR, quelle que soit la forme sous laquelle il se manifeste, est accompagné de reformulations : en ( I I), la reformulation précède le DI; en (IO) et en (I2) la reformulation suit un DD. L'ensemble de ces formes est intéressant, du point de vue de leur prise en charge dans la co-construction des explications, permettant au locuteur citant de marquer et de graduer son accord ou son désaccord, par un jeu complexe relevant de la «concordance discordante» ou de la « discordance concordante» (cf. infra, 3. pour la définition de ces notions).

\subsection{Lieu d'émergence du DR dans la structure de l'explication}

Le DR peut se manifester dans les trois phases de la séquence (Coltier, de Gaulmyn), comme dans les schématisations initiales ou finales. D'une manière générale, le DR joue un rôle pragmatique de cadre initial posant l'explanandum ou de balise finale reformulant l'explanans (Lund 2003 : I 29, I69). Dans la phase initiale, le DR exprime soit une schématisation collective du problème à expliquer, soit une ou plusieurs schématisations personnelles. Il s'agit alors d'activer le processus explicatif, aussi le DR donne-t-il volontiers à la schématisation un tour excessif ou distancié, comme en (I I), de façon à permettre une réaction positive des interlocuteurs, qui les mette en confiance pour approfondir l'explication. Une autre stratégie consiste à citer de façon initiale deux DR exprimant des schématisations antagonistes, comme en (9) et (Io) ou dans le deuxième tour de parole de l'exemple (I 2).

On retrouve également du DR à des moments charnières de l'explication, tant sur le plan interactionnel que cognitif. Une de ses fonctions vise à régler une difficulté : ainsi les DR antagonistes du deuxième tour de parole de (I2) sont-ils réduits à leur plus simple expression («oui » ou «non »), l'essentiel étant ensuite de s'interroger (« pourquoi ») et de « donner des arguments». Cette amorce de la réflexion est relancée par le maître qui sollicite « un autre avis que 
Loïc et pourquoi » et qui insiste à plusieurs reprises sur la nécessité de donner des arguments, en reprenant la matrice antithétique initiale : "Alors un astéroïde et un météorite c'est la même chose? Qui sont d'accord avec Etienne et pourquoi», «Qui n'est pas d'accord et pourquoi».

Le DR n'est pas l'apanage du maître, même si ce dernier en use plus souvent que les élèves. En ( I 2), le DR apparaît chez un élève au cœur du parcours explicatif, lorsqu'un des élèves (EI) argumente en faveur de la différence entre astéroïde et météorite à partir de l'existence significative et motivée de deux dénominations différentes : «Ben oui parce que ils auraient pas appelé un, un...». Ainsi, la fonction dialogique du DR est un solide point d'appui linguistique et énonciatif pour construire deux PDV différents sur l'objet à partir desquels il est pensable de mieux expliquer.

Le DR sert également à ratifier ou au minimum à dresser un bilan provisoire; dans ce cas, le DR prend la forme d'un point de vue collectif que le locuteur citant partage plus ou moins, comme on le verra en (I3) et (I4) :

(13) (Corpus «Machin mouillé»)

M [alors] on dirait que quand c'est au pluriel $\backslash$ oo on n'aurait pas de s / oo et que quan[t] c'est au singulier/ on aurait un $\mathrm{s} \backslash$ c'est ça qu'vous dites $\backslash$ en fait $\backslash$

(I4) (Corpus « Machin mouillé »)

$\mathrm{M}$ on a dit que : la troisième personne du singulier ça s`rait avec un s/ o et à la troisième personne du pluriel on n'en aurait pas besoin $\backslash$ oo mais c'est quand même bizarre / là on a toujours notre problème / avec cette histoire de cheveux / hein /

Enfin, la fonction de ratification par le DR apparaît en fin d'explication. On peut s'étonner que la ratification passe par du DR : comme on le verra en (I6), le DR repose sur une explication/ argumentation par autorité polyphonique, évoquant le discours du Savoir, éventuellement en incluant les élèves dans le discours savant, grâce à un nous ou un on qui leur confère ainsi un brevet de «bons explicants », comme on le verra en (I6).

Tous ces phénomènes de bivocalité sont si complexes qu'ils requièrent, pour être pleinement analysés, les concepts de surénonciation ou de sousénonciation. Ainsi, en (I5), le DI est d'emblée une reformulation prise en charge par le maître, qui, en tant que 
surénonciateur, oriente le dit des élèves vers la comparaison avec les dinosaures; cette posture de surplomb est encore plus nette avec la reformulation suivante, qui explicite le sens de la reformulation précédente, ainsi qu'avec l'enchaînement du maître («ce qui fait que...»), qui exprime un argument par autorité polyphonique :

(I 5) (Corpus «Dinosaures »)

M non / alors là j'en étais sûr hein vous avez pas bien entendu \o y sont en train de dire / o que o il est intéressant d'étudier comment vivent les iguanes marins I parce que o ils ont les MEmes caractéristiques o que les dinosaures qui ont disparu $\backslash$ o ce qui fait que si on les étudie bien eux / o on va avoir o une idée o de la façon o dont vivaient les dinosaures /

\section{La fonction des discours représentés dans le type de texte explicatif : surénonciation, sousénonciation, entre concordance et discordance}

Les diverses formes d'hétérogénéité constitutive et montrée participent de la discordance énonciative, tout en ayant la capacité de construire des formes et degrés variés de concordance cognitive et interactionnelle. En contexte interactionnel, la concordance est un phénomène instable et limité dans l'espace et dans le temps (Rabatel 2005, 2006), c'est pourquoi il y a lieu de distinguer la "concordance concordante »- si l'on ose une tournure qui n'a de pléonastique que l'apparence - de la "concordance discordante »; et de même pour la discordance. Autrement dit, la représentation habituelle des interactions consensuelles ou dissensuelles est trop platement binaire, alors que les interactions reposent sur un continuum, pour lequel nous nous inspirerons librement des notions de « concordance discordante » et de « discordance concordante» de Ricœur dans Temps et récit, pour signifier que, dans le cas d'une logique de co-construction d'un objet de discours (en l'occurrence un explanandum ou un explanans), le processus de co-construction est dominé, outre les phases de consensus et de dissensus, par deux phases intermédiaires caractérisées :

- tantôt par un mouvement dominant centripète de convergence des deux locuteurs / énonciateurs pour construire l'objet (concordance), lequel mouvement intègre néanmoins une 
posture en surplomb d'un des deux énonciateurs qui affirme sa prééminence dans la co-construction, en sorte que la concordance fait entendre une discordance;

- tantôt par un mouvement dominant centrifuge de désaccord des deux locuteurs/énonciateurs (discordance), qui n'ose pourtant aller à terme, par le fait que l'un des énonciateurs rapporte un PDV antérieur en le bémolisant (cf. le locuteur vicariant de Danon-Boileau et Morel 2003: 242), en sorte que la discordance en cours émerge de la prise de distance envers un accord antérieur :

Partant de là, on propose de définir la concordance concordante comme la seule forme de coénonciation véritable; la concordance discordante comme surénonciation, la discordance concordante comme sousénonciation. Seule la coénonciation relève d'une colocution qui va jusqu'à la prise en charge énonciative d'un PDV commun, tandis que la sousénonciation, comme la surénonciation, reposent sur une co-construction d'un seul PDV dans un topic de séquence ${ }^{\mathrm{I}}$, sans que les deux locuteurs ne s'engagent autant l'un que l'autre, soit que le surénonciateur impose son PDV à l'autre, en faisant comme si son PDV n'était qu'une paraphrase de celui de l'interlocuteur, soit que le sousénonciateur reprenne le PDV de l'autre, en s'en distanciant sans aller néanmoins jusqu'à lui substituer un PDV antagoniste. Bref, ces deux dernières postures indiquent une dissymétrie dans la co-construction d'un contenu propositionnel plus ou moins assumé par les deux locuteurs à travers ses reprises et reformulations, tandis que la discordance discordante relève de l'expression manifeste et explicite de deux PDV antinomiques.

I. Le topic est défini ici moins comme l'information donnée (Givòn I995), saillante (Ariel I988) que comme l'information à propos de laquelle quelque chose est prédiqué (Lambrecht 1994) et qui organise une séquence « conversationnelle » (Rabatel 2004) dans un contexte interactionnel (Mondada 2003). 
$\begin{array}{ccc}\text { Concordance concordante concordance discordante } & \text { discordance concordante discordance discordante } \\ \downarrow & \downarrow & \downarrow \\ \text { Coénonciation d'1 PDV surénonciation d'1 PDV sousénonciation d'1 PDV énonciation de 2 PDV opposés } \\ \text { Consensus } \longleftrightarrow \text { dissensus }\end{array}$

Compte tenu de l'importance des interactions dissymétriques, et, dans ce cadre, des phases intermédiaires de co-construction des PDV, on se bornera aux formes de concordance discordante et de discordance concordante.

\section{I. Surénonciation et concordance discordante}

En (I6), I02 est une reformulation non paraphrastique de 99 et IOI, incluant un DI qui joue un rôle charnière dans l'argumentation par autorité polyphonique.

(I6) (Corpus « Polyèdres »)

$94 \mathrm{M} \quad$ (montre un solide : prisme droit à base triangulaire) ce genre/ oo de polyèdre \est-ce que c'est un polyèdre REgulier \

95 Es non::

$96 \mathrm{M}$ pourquoil

97 E parce que :

98 M Arnaud

99 ARN parce que c'est pas les:: les mêmes euh:: les mêmes euh figures qu'ils ont qu'ils ont utilisées pour euh::

I $00 \mathrm{M}$ ils ont utilisé pour le faire o des $\backslash$

IOI Es triangles $\backslash$

I02 M des triangles et des RECtangles $\backslash$ pour que ce soit un polyèdre régulier on vous dit que toutes les FAces doivent être Identiques $\backslash o$ en forme lo et en taille $\backslash$ o donc CA / o ce n'est pas un polyèdre régulier \ donc quand je hein /

L'explanandum ne donne pas aisément naissance à des explications claires, tant sur le plan langagier que cognitif; certains sont capables de répondre, mais peinent à expliquer. Arnaud s'y essaye en 99, par un énoncé en parce que qui complète sémantiquement la question du maître en 94 et, syntaxiquement, la réponse du tour 95.

La comparaison de 99 et 102 révèle de notables différences. Le remplacement de "parce que » par "pour que » (qui ne sont pas au même niveau syntaxique), les changements de temps et de mode 
et de lexique font de I02 un énoncé générique prescriptif (d'où la modalité déontique associée aux énoncés, qui contraint les opérations intellectuelles) invitant à vérifier si ce polyèdre possède les traits définitoires de la classe des polyèdres réguliers :

Parce que $\rightarrow$ pour que... donc

$\varnothing \rightarrow$ on vous dit que

C'est $\rightarrow$ ce soit

Qu'ils ont utilisées $\rightarrow$ doivent être identiques en forme et en taille

Figures $\rightarrow$ faces

I02 est une phrase multiple, fournissant l'occasion d'un «petit drame » mettant en scène les données du raisonnement explicatif à mettre en œuvre : la subordonnée équivaut à une question qui trouve sa réponse en deux temps, d'abord dans une principale (assortie de sa complétive « on vous dit que », qui évoque des conditions nécessaires), ensuite dans la coordonnée introduite par « donc », qui conclut en fonction de l'absence des traits définitoires des polyèdres réguliers.

Sur le plan énonciatif, grâce au «on », le maître se pose comme un expert qui s'appuie sur l'autorité du Savoir scientifique. Théoriquement, la valeur indéfinie $d u$ « on » pourrait inclure les apprenants - qui au demeurant, sont loin d'être un bloc indivisible mais ces derniers sont situés à ce moment de l'interaction et de la réflexion en extériorité et en position dominée («on vous dit»), ce qui fait ressortir la position surplombante du maitre. Le DI joue ici un rôle d'argument par autorité polyphonique ${ }^{\mathrm{I}}$, comme si le fait d'avoir été prononcé par une référence incontestable renforçait le statut intrinsèque de l'explanans.

On a là un ensemble de traits linguistiques qui ne donnent pas au locuteur de I02 la même posture énonciative que celle de 99,

I. Il y a argument par autorité polyphonique lorsque, à propos d'une proposition $P$, le locuteur, d'une part indique que $P$ a déjà été, est actuellement, ou pourrait être l'objet d'une assertion et «présente ce fait comme donnant de la valeur à la proposition $P$, comme la renforçant, comme lui ajoutant un poids particulier » (Ducrot I984: I 50); d'autre part appuie sur cette première assertion une seconde assertion, relative à une autre proposition, $Q$ (ibid. : I 54), le locuteur manifestant l'existence d'un lien légitime (sinon nécessaire) entre l'acceptation de $P$ et celle de $Q$. 
et qu'il est légitime de nommer surénonciation dans la mesure où, même s'il semble dire en apparence la " même » chose, il confère à son énoncé un statut dominant sur le plan cognitif, sur le plan de l'activité, comme sur le plan linguistique. Il y a donc une concordance dans la mesure où le maître s'appuie factuellement sur le discours d'Arnaud, mais celle-ci est discordante car la visée explicative est plus abstraite et plus générale, plus facilement transposable. On retrouve cette concordance discordante dans l'exemple suivant :

\begin{tabular}{|c|c|}
\hline (I7) & (Corpus « Polyèdres ») \\
\hline I I $6 \mathrm{M}$ & $\begin{array}{l}\text { (montre un solide: un tétraèdre) est-ce que ça c'est un polyèdre régu- } \\
\text { lier } \backslash\end{array}$ \\
\hline I 77 Es & oui:: \\
\hline I I $8 \mathrm{M}$ & $\begin{array}{l}\text { il répond bien effectivement o aux QUAtre règles } \backslash \text { oo est-ce que CA } \\
\text { (montre un cube) c'est un polyèdre régulier } \backslash\end{array}$ \\
\hline I I9 Es & oui:: \\
\hline $\mathrm{I} 20 \mathrm{M}$ & il répond aux quatre règles $\backslash$ o JE VOIS Teddy qui= \\
\hline I2I TED & =j'ai pas fini \\
\hline I $22 \mathrm{M}$ & bah oui mais $\backslash$ o pourquoi je vais lui dire d'arrêter Teddy $\backslash$ \\
\hline I $23 \mathrm{E}$ & parce que ça a pas la même longueur $\backslash$ \\
\hline $\mathrm{I} 24 \mathrm{M}$ & $\begin{array}{l}\text { c'est pas un o POlygone régulier \o lui il n'a pas les côtés de la même } \\
\text { longueur donc des rectangles déjà euh : oo on peut dire qu'on va } \\
\text { les laisser tomber } \backslash \text { oo Alors je vous ai arrêtés parce que maintenant } \\
\text { il va falloir passer à la DEUxième partie de la consigne / o on en a } \\
\text { trouvé des vous avez à peu près tous trouvé un ou deux polyèdres } \\
\text { réguliers / o mais maintenant je vais vous demander de les trouver o } \\
\text { TOUS / }\end{array}$ \\
\hline
\end{tabular}

La concordance discordante repose sur le fait qu'en i 24 le maître reformule de façon plus abstraite les explications du tour précédent - notions géométriques (« rectangle» vs carré) clarifiant la notion de «longueur»- et reprend la question initiale de I I6. Le dit et le dire visent à habituer les élèves à une démarche intellectuelle confrontant systématiquement les critères de la classe des polyèdres réguliers avec la diversité des solides. La reformulation intervient en fin d'explication pour faire partager la ratification par les élèves, érigés ici en membres à part entière de la communauté savante (à la différence de (I6)). Leur rôle n'est pas seulement conclusif, il est aussi initiatif, puisque la clôture de l'épisode est une des conditions nécessaires pour passer à une phase ultérieure. Interactionnellement, le maître fait donc comme si les élèves, par leur compréhension, l'autorisaient à passer à la "deuxième partie de la consigne». On se 
trouve face à un coup de force ${ }^{\mathrm{I}}$ vertueux, tant sur les plans langagier que cognitif et pédagogique, confirmant la posture surplombante du maître.

Qu'est-ce donc qui fait surénonciation ici, à travers l'utilisation du DR et des reformulations par le maître? Sur le plan cognitif, le maître est surénonciateur parce qu'il reprend des PDV d'élèves justes, tout en les reformulant d'une façon plus abstraite, en explicitant les étapes du raisonnement. Ce PDV feint d'être la reformulation pure et simple du précédent, mais, en réalité, il va plus loin que le PDV des élèves, en se situant à un niveau plus englobant. Sur le plan linguistique, la surénonciation se marque à travers des faisceaux de marques congruentes : redénominations apparemment synonymiques, changement de temps, de modalité, trame plus serrée de connecteurs, rôle du DR (réel ou putatif) et des reformulations qui attribuent des PDV aux élèves, tout en les reformulant dans un sens plus général et en s'appuyant sur le discours de la Science, dans une argumentation par autorité polyphonique. Sur le plan interactionnel, enfin, le maître oriente les activités passées ou futures, en étant responsable non seulement du déroulement des activités (passage à un autre explanandum, changement de séquence, etc.), mais en leur donnant un sens.

\subsection{Sousénonciation et discordance concordante}

Ces critères se retrouvent comme inversés dans la sousénonciation : ainsi le maître, en (I8), utilise un DD écho à distance sous une forme dialogique exacerbée pour relancer la discussion, sans partager ce PDV, mais pour faire émerger par contraste un PDV commun - (4) présente la même tactique - en quoi la discordance énonciative initiale est finalement concordante, du fait de son objectif de mettre les élèves sur la voie d'une explication commune. Et sa reformulation dans le tour 7 s'appuie sur les énoncés antérieurs des élèves. Certes, l'énoncé est plus construit, plus explicite, mais il se présente comme le même contenu propositionnel que celui des élèves. Qui plus est, la formulation ne fait que reformuler sans

I. En dépit du caractère apparemment non impositif du modal « on peut dire qu'on pourrait », la réduplication équivaut contextuellement à un « devoir », en raison de l'absence des traits définitoires de la classe des polyèdres réguliers. 
ouvrir sur un nouvel espace de problématisation, qui proviendra des élèves, avec la question de la preuve et de son écriture ${ }^{\mathrm{I}}$.

(I8) (Corpus «OVNI $»)$

I M non non:: vous allez produire un compte rendu tout seuls /

2 Es ((brouhaha))

3 M alors là on se recentre/ oo bon Nadège tu veux dire quelque chose toi $\backslash \mathrm{o}$ alors tu nous le redis bien fort /

4 NAD bah (inaud.) pour le débat/

$5 \mathrm{M}$ donc tu disais le débat il sert à rien parce que / o y'en a qui n'ont:: personne : à ton avis personne n'a changé d'avis hein c'est ça

6 Es Plusieurs élèves manifestent, certains affirmant qu'ils ont changé d'avis, d'autres non

7 M alors autrement dit / o le débat il a peut-être servi / o à voir les deux:: aspects de la question $\ o$ avant de commencer / o vous aviez / la majorité hein \vous aviez peut-être o une seule idée hein \pour / o ou seulement l'idée contre / o et finalement peut-être que le débat il a servi à ça o à voir les deux points de vue $\backslash$ oo Oui $\backslash$

8 E : ceux qui sont contre/ o y peuvent pas dire que ça existe pas parce que ils ont:: ils peuvent pas le prouver/ pis ceux qui sont pour y peuvent pas prouver que ça existe /

9 M voilà, le problème c'est les preuves hein / odans ce sujet là $\ 0$ effectivement $\backslash$

Ainsi, sur le plan cognitif comme sur le plan linguistique, le sousénonciateur reprend le PDV des interactants, même s'il le met à distance : il est toujours question de deux PDV, mais alors qu'initialement les élèves envisageaient deux PDV de deux énonciateurs différents sur un seul objet, il est question de "deux aspects de la question » qui renvoient à «deux points de vue» sans que soit tranché l'ancrage énonciatif du futur compte rendu (deux locuteurs / énonciateurs distincts ou un seul). La triplication des «peutêtre », qui scandent la dimension hypothétique de la reformulation, épouse empathiquement le mouvement de prise de conscience progressive des élèves. On voit que le maître se met à la place des élèves, sans aller jusqu'aux coups de force précédents dans sa démarche explicative, puisque, sur le plan interactionnel, c'est un autre élève

I. C'est donc un élève (et non le maître), en 8, qui adopte une posture de surénonciation envers ses autres camarades. Un des relecteurs se demande si le but ultime de la pédagogie (transmettre aux élèves l'assurance et la maîtrise) passe par l'appropriation d'une posture de surénonciateur. C'est vrai dans ce contexte; mais le raisonnement ne doit pas être généralisé sans précaution, car il est possible d'imposer à ses interlocuteurs des PDV surénoncés cognitivement absurdes.. 
qui ouvre une nouvelle séquence consacrée à la question de la preuve et à celle, conjointe, de la formulation des hypothèses.

Nous conclurons sur ce que la saisie du DR, du dialogisme et des interactions orales apporte à la compréhension de l'explication. En premier lieu, insistons, après d'autres, sur la productivité de la notion de parcours dans des explications en contexte didactique. En deuxième lieu, soulignons que si l'explication repose sur une autorité, cette dernière se (re)joue constamment dans l'interaction : c'est pourquoi elle ne dépend pas que du seul statut externe des individus, comme le montrent les changements de posture énonciative du maître et des élèves. En troisième lieu, retenons que, au cœur du parcours explicatif, la co-construction interactionnelle des explications est instable, dissymétrique, sur les plans cognitifs et énonciatifs, sans que pour autant les changements de PDV sur l'objet ou les désaccords ne fassent échouer l'explication. Au contraire, c'est par la maîtrise de dissensus tempérés que l'explication se construit et que les sujets s'en pénètrent, d'où le rôle stratégique des mécanismes de concordance discordante et de discordance concordante. Ces derniers ne sont certes pas réservés à l'explication, pas plus qu'ils ne sont impliqués par elle, mais ils jouent un rôle positif dans la gestion de l'interaction et dans la résolution / formulation des explications en s'aidant de la diversité énonciative et la faisant converger vers la résolution du problème.

En quatrième lieu, et c'est sans doute à nos yeux la conclusion la plus innovante, insistons sur le fait que l'analyse des processus explicatifs en contexte dialogal modifie qualitativement le dialogisme. En effet, en contexte monologal, le dialogisme revient le plus souvent, pour le locuteur, à anticiper sur les désaccords possibles du destinataire du message pour mieux le convaincre : en ce sens, le destinataire est érigé, comme chez Culioli, en un coénonciateur qui n'a pas d'existence autonome, et qui n'existe que par les représentations intersubjectives que le locuteur se fait de l'autre. En contexte dialogal, les stratégies discursives des locuteurs / énonciateurs sont autonomisées et chaque locuteur fait jouer dans sa propre voix des PDV variés - qu'il s'agisse des siens, de ceux d'un interlocuteur, d'un tiers ou de la communauté, de PDV putatifs ou contrefactuels, etc. - dont il est plus ou moins proche et qui lui permettent de 
co-construire des explications ${ }^{\mathrm{I}}$. Les phénomènes auto- ou hétérodialogiques ne se limitent pas à l'instrumentalisation du PDV des destinataires à partir d'un vouloir dire bien défini, puisque le processus explicatif ne repose pas sur l'actualisation d'un déjà dit explicatif antérieur, mais sur l'élaboration d'un à dire et d'un dire qui émergent progressivement au fil du discours et de l'interaction.

\section{Références bibliographiques}

Adam J.-M. $\quad 2005$ La linguistique textuelle. Armand Colin, Paris.

Ariel M. $\quad$ I 988 «Referring and accesssibility », Journal of pragmatics, 24, 65-87.

Bres J. et Verine B. 2002 «Le bruissement des voix dans le discours : dialogisme et discours rapporté », Faits de Langues, I9, I 59I69.

Bronckart J.-P. I 997 Activité langagière, textes et discours. Delachaux et Niestlé, Lausanne, Paris.

Bruxelles S. et de Gaulmyn M.-M.

200 I « Explication en interaction : facteurs déterminants et degré d'efficacité », Psychologies de l'interaction, V 9-10, 47-75

Colletta J.-M. 2004 Le développement de la parole chez l'enfant de 6 à II ans. Corps, langage et cognition. Mardaga, Sprimont.

Coltier D. I986 «Approches du texte explicatif», Pratiques 5I, 3-22.

Danon-Boileau L. et Morel M.-A. 2003 « Le locuteur vicariant », in Merle, J.-M. (éd), Le sujet, 235-246. Ophrys, Gap, Paris.

De Gaulmyn, M.-M.

I99I, "Les cinq types de questions dans l'explication conversationnelle», in Kerbrat-Orecchioni C. (éd), La question, 295-322. Presses universitaires de Lyon, Lyon.

De Gaulmyn, M.-M., Bouchard, R., et Rabatel, A. (éds) $200 \mathrm{I}$ Le Processus rédactionnel. Écrire à plusieurs voix. L'Harmattan, Paris.

I. Dans ces processus, rappelons l'importance de la gestualité paraverbale (Colletta $2004: 245^{-252}$ ). 
Ducrot O. $\quad$ I984 Le dire et le dit. Minuit, Paris.

Ebel M. I98I « L'explication : acte de langage et légitimité du discours ", Revue européenne des sciences sociales. Cahiers Vilfredo Pareto, t. XIX, 56, I 5-36.

Givòn T. et al. I 995 Coherence in spontaneous speech. John Benjamins Publishing Company, Amsterdam/Philadelphia.

Grize J.-B. I98 I «Logique naturelle est explication », Revue européenne des sciences sociales. Cahiers Vilfredo Pareto, t. XIX, 56, 7-I4.

Grize J.-B. $\quad$ I990 Logique et langage. Ophrys, Gap, Paris.

Guernier, M.-C., Durand-Guerrier, V. et Sautot, J.-P. (éds) 2006 Interactions verbales, didactiques et apprentissages. Recueil, traitement et interprétation didactiques des données langagières en contextes scolaires, Besançon : Presses universitaires de Franche-Comté.

Joab M., Rossari C.

I 999 « Analyse automatique de séquences explicatives fondée sur le modèle genevois d'analyse du discours ", Psychologies de l'interaction, V 9, 26I-288.

Lambrecht K. I994 Information structure and sentence form : topic, focus and the mental representation of discourse referents. Cambridge University Press, Cambridge.

Lepoire S. I $\quad$ I 999 «Aspects cognitifs de la conduite d'un dialogue explicatif », Pratiques, I03-I04, I73-I92.

Lund K. 2003 Analyse de l'activité explicative en interaction. Thèse de doctorat, nouveau régime, université de Grenoble I.

Martin F. 2005 Les prédicats d'état et d'achèvement en discours. Sémantique des prédicats évaluatifs et psychologiques. Thèse de doctorat, Université libre de Bruxelles.

Miéville D. $\quad$ I98 I « Explication et discours didactique de la mathématique », Revue européenne des sciences sociales. Cahiers Vilfredo Pareto, t. XIX, 56, I I 5-I 52.

Moirand S. $\quad 2002$ Article «Explication et transmission de connaissances », in Charaudeau P. et Maingueneau D. (éds) Dictionnaire d'analyse $d u$ discours, 255-256. Le Seuil, Paris. 
Mondada L. $\quad 2003$ «Parler topical et organisation séquentielle : 1'apport de l'analyse conversationnelle », Verbum, XXV, I 93-2I9.

Morf A. et Leclerc S.

I98 I « Les aspects cognitifs du discours d'explication ", Travaux du centre de recherches sémiologiques, 36, 83-93.

Nølke H., Fløttum K. et Norén C. 2004 Scapoline. La théorie scandinave de la polyphonie linguistique. Kimé, Paris.

Plantin C. $\quad 2002$ Article «Explication », in Charaudeau P. et Maingueneau D. (éds) Dictionnaire d'analyse du discours, 25I-254. Le Seuil, Paris.

Rabatel A. $\quad 2000$ "Valeurs représentative et énonciative du "présentatif" c'est et marquage du point de vue ", Langue Française, I28, 52-73.

Rabatel A. 200I «Valeurs énonciative et représentative des "présentatifs" c'est, il y a, voici / voilà : effet point de vue et argumentativité indirecte du récit », Revue de Sémantique et Pragmatique, 9-I0, 43-74.

Rabatel A. $\quad 2002$ «Le sous-énonciateur dans les montages citationnels : hétérogénéités énonciatives et déficits épistémiques », Enjeux, 54, 52-66.

Rabatel A. $\quad 2003$ «Les verbes de perception en contexte d'effacement énonciatif : du point de vue représenté aux discours représentés », Travaux de linguistique, 46, 49-88.

Rabatel A. 2004a « Déséquilibres interactionnels et cognitifs, postures énonciatives et co-construction des savoirs : coénonciateurs, sur-énonciateurs et archi-énonciateurs "), in Interactions orales en contexte didactique. Mieux (se) comprendre pour mieux (se) parler et pour mieux ( $s^{\prime}$ ) apprendre, Rabatel, A. (éd.), 29-66. Presses Universitaires de Lyon, Lyon.

Rabatel A. $\quad 2004$ b « L'effacement énonciatif dans les discours rapportés et ses effets pragmatiques », Langages, I 56, 3-I 7 .

Rabatel A. $\quad 2004 \mathrm{c}$ «Stratégies d'effacement énonciatif et surénonciation dans le Dictionnaire philosophique de ComteSponville », Langages, I 56, I 8-33. 
Rabatel A. $\quad 2004$ « Faciliter l'appropriation de la réflexion métalinguistique au cycle 3 », Cahiers du Français contemporain, 9, I4 I-I 53 .

Rabatel A. $\quad 2005$ «Les postures énonciatives dans la co-construction dialogique des points de vue : coénonciation, surénonciation, sousénonciation », in Bres, J., Haillet, P.P., Mellet, S., Nolke, H., Rosier, L., (éds.). Dialogisme, polyphonie : approches linguistiques, 95-I Io. Duculot, Bruxelles.

Rabatel A. $\quad 2006$ «Stratégies discursives de concordance discordante ou de discordance concordante dans les reformulations en contexte didactique », Colloque international Voies de la reformulation, Rennes, I 9 et 20 mai 2006.

Ricœur P. I983 Temps et récit, t. I. Le Seuil, Paris.

Rosier L. $\quad$ I999 Le discours rapporté. Duculot, Bruxelles.

Saussure L. de 2003 Temps et pertinence. DeBoeck-Duculot, Bruxelles. Schneuwly B. et Rosat M.-C.

I986 «Analyse ontogénétique des organisateurs textuels dans deux textes informatifs écrits », Pratiques 5I, 39-53.

Vincent D. et Dubois S.

I 997 Le discours rapporté au quotidien. Nuit Blanche Éditeur, Québec. 